\title{
Search for a low-mass scalar Higgs boson decaying to a tau pair in single-photon decays of $\mathbf{Y}(1 S)$
}

J. P. Lees, ${ }^{1}$ V. Poireau, ${ }^{1}$ V. Tisserand,${ }^{1}$ J. Garra Tico, ${ }^{2}$ E. Grauges, ${ }^{2}$ A. Palano, ${ }^{3 a, 3 b}$ G. Eigen, ${ }^{4}$ B. Stugu, ${ }^{4}$ D. N. Brown,${ }^{5}$ A. Georges, ${ }^{5}$ L. T. Kerth, ${ }^{5}$ Yu. G. Kolomensky, ${ }^{5}$ G. Lynch,${ }^{5}$ U. Paudel,${ }^{5}$ H. Koch, ${ }^{6}$ T. Schroeder, ${ }^{6}$ D. J. Asgeirsson, ${ }^{7}$ C. Hearty, ${ }^{7}$ T. S. Mattison, ${ }^{7}$ J. A. McKenna, ${ }^{7}$ R. Y. So, ${ }^{7}$ A. Khan, ${ }^{8}$ V. E. Blinov, ${ }^{9}$ A. R. Buzykaev, ${ }^{9}$ V. P. Druzhinin, ${ }^{9}$ V. B. Golubev, ${ }^{9}$ E. A. Kravchenko, ${ }^{9}$ A. P. Onuchin, ${ }^{9}$ S. I. Serednyakov, ${ }^{9}$ Yu. I. Skovpen, ${ }^{9}$ E. P. Solodov, ${ }^{9}$ K. Yu. Todyshev, ${ }^{9}$ A. N. Yushkov, ${ }^{9}$ M. Bondioli, ${ }^{10}$ D. Kirkby,${ }^{10}$ A. J. Lankford,,${ }^{10}$ M. Mandelkern,,${ }^{10}$ H. Atmacan, ${ }^{11}$ J. W. Gary, ${ }^{11}$ F. Liu, ${ }^{11}$ O. Long, ${ }^{11}$ G. M. Vitug, ${ }^{11}$ C. Campagnari, ${ }^{12}$ T. M. Hong, ${ }^{12}$ D. Kovalskyi, ${ }^{12}$ J. D. Richman, ${ }^{12}$ C. A. West, ${ }^{12}$ A. M. Eisner, ${ }^{13}$ J. Kroseberg, ${ }^{13}$ W. S. Lockman, ${ }^{13}$ A. J. Martinez, ${ }^{13}$ B. A. Schumm, ${ }^{13}$ A. Seiden, ${ }^{13}$ D. S. Chao, ${ }^{14}$ C. H. Cheng, ${ }^{14}$ B. Echenard, ${ }^{14}$ K. T. Flood, ${ }^{14}$ D. G. Hitlin, ${ }^{14}$ P. Ongmongkolkul, ${ }^{14}$ F. C. Porter, ${ }^{14}$ A. Y. Rakitin, ${ }^{14}$ R. Andreassen, ${ }^{15}$ Z. Huard, ${ }^{15}$ B. T. Meadows, ${ }^{15}$ M. D. Sokoloff, ${ }^{15}$ L. Sun, ${ }^{15}$ P. C. Bloom, ${ }^{16}$ W. T. Ford,${ }^{16}$ A. Gaz ${ }^{16}$ U. Nauenberg, ${ }^{16}$ J. G. Smith, ${ }^{16}$ S. R. Wagner, ${ }^{16}$ R. Ayad,${ }^{17, \dagger}$ W. H. Toki, ${ }^{17}$ B. Spaan,${ }^{18}$ K. R. Schubert ${ }^{19}$ R. Schwierz, ${ }^{19}$ D. Bernard, ${ }^{20}$ M. Verderi, ${ }^{20}$ P. J. Clark, ${ }^{21}$ S. Playfer, ${ }^{21}$ D. Bettoni, ${ }^{22 a}$ C. Bozzi, ${ }^{22 a}$ R. Calabrese, ${ }^{22 a, 22 b}$ G. Cibinetto, $, 2 a, 22 b$ E. Fioravanti, ${ }^{22 a, 22 b}$ I. Garzia, ${ }^{22 a, 22 b}$ E. Luppi, ${ }^{22 a, 22 b}$ M. Munerato,${ }^{22 a, 22 b}$ L. Piemontese, ${ }^{22 a}$ V. Santoro, ${ }^{22 a}$ R. Baldini-Ferroli, ${ }^{23}$ A. Calcaterra, ${ }^{23}$ R. de Sangro, ${ }^{23}$ G. Finocchiaro, ${ }^{23}$ P. Patteri, ${ }^{23}$ I. M. Peruzzi, ${ }^{23, \sharp}$ M. Piccolo, ${ }^{23}$ M. Rama, ${ }^{23}$ A. Zallo, ${ }^{23}$ R. Contri, ${ }^{24 a, 24 b}$ E. Guido, ${ }^{24 a, 24 b}$ M. Lo Vetere, ${ }^{24 a, 24 b}$ M. R. Monge, ${ }^{24 a, 24 b}$ S. Passaggio, ${ }^{24 a}$ C. Patrignani, ${ }^{24 a, 24 b}$ E. Robutti, ${ }^{24 a}$ B. Bhuyan, ${ }^{25}$ V. Prasad,${ }^{25}$ C. L. Lee, ${ }^{26}$ M. Morii, ${ }^{26}$ A. J. Edwards,${ }^{27}$ A. Adametz, ${ }^{28}$ U. Uwer, ${ }^{28}$ H. M. Lacker, ${ }^{29}$ T. Lueck, ${ }^{29}$ P. D. Dauncey, ${ }^{30}$ U. Mallik, ${ }^{31}$ C. Chen, ${ }^{32}$ J. Cochran, ${ }^{32}$ W. T. Meyer, ${ }^{32}$ S. Prell, ${ }^{32}$ A. E. Rubin, ${ }^{32}$ A. V. Gritsan, ${ }^{33}$ Z. J. Guo, ${ }^{33}$ N. Arnaud, ${ }^{34}$ M. Davier, ${ }^{34}$ D. Derkach, ${ }^{34}$ G. Grosdidier, ${ }^{34}$ F. Le Diberder, ${ }^{34}$ A. M. Lutz, ${ }^{34}$ B. Malaescu, ${ }^{34}$ P. Roudeau, ${ }^{34}$ M. H. Schune, ${ }^{34}$ A. Stocchi, ${ }^{34}$ G. Wormser, ${ }^{34}$ D. J. Lange, ${ }^{35}$ D. M. Wright, ${ }^{35}$

C. A. Chavez, ${ }^{36}$ J. P. Coleman, ${ }^{36}$ J. R. Fry, ${ }^{36}$ E. Gabathuler, ${ }^{36}$ D. E. Hutchcroft, ${ }^{36}$ D. J. Payne, ${ }^{36}$ C. Touramanis, ${ }^{36}$

A. J. Bevan, ${ }^{37}$ F. Di Lodovico, ${ }^{37}$ R. Sacco, ${ }^{37}$ M. Sigamani, ${ }^{37}$ G. Cowan ${ }^{38}$ D. N. Brown, ${ }^{39}$ C. L. Davis,${ }^{39}$ A. G. Denig,${ }^{40}$ M. Fritsch, ${ }^{40}$ W. Gradl,${ }^{40}$ K. Griessinger ${ }^{40}$ A. Hafner, ${ }^{40}$ E. Prencipe, ${ }^{40}$ R. J. Barlow, ${ }^{41,}$ G. Jackson, ${ }^{41}$ G. D. Lafferty, ${ }^{41}$ E. Behn, ${ }^{42}$ R. Cenci, ${ }^{42}$ B. Hamilton, ${ }^{42}$ A. Jawahery, ${ }^{42}$ D. A. Roberts, ${ }^{42}$ C. Dallapiccola, ${ }^{43}$ R. Cowan, ${ }^{44}$ D. Dujmic ${ }^{44}$

G. Sciolla, ${ }^{44}$ R. Cheaib, ${ }^{45}$ D. Lindemann, ${ }^{45}$ P. M. Patel, ${ }^{45}$, S. H. Robertson, ${ }^{45}$ P. Biassoni, ${ }^{46 a, 46 b}$ N. Neri, ${ }^{46 a}$

F. Palombo, ${ }^{46 a, 46 b}$ S. Stracka, ${ }^{46 a, 46 b}$ L. Cremaldi, ${ }^{47}$ R. Godang, ${ }^{47, \|}$ R. Kroeger, ${ }^{47}$ P. Sonnek, ${ }^{47}$ D. J. Summers, ${ }^{47}$

X. Nguyen,${ }^{48}$ M. Simard ${ }^{48}$ P. Taras,${ }^{48}$ G. De Nardo, ${ }^{49 a, 49 b}$ D. Monorchio, ${ }^{49 a, 49 b}$ G. Onorato, ${ }^{49 a, 49 b}$ C. Sciacca, ${ }^{49 a, 49 b}$ M. Martinelli, ${ }^{50}$ G. Raven, ${ }^{50}$ C. P. Jessop, ${ }^{51}$ J. M. LoSecco, ${ }^{51}$ W. F. Wang,,${ }^{51}$ K. Honscheid,${ }^{52}$ R. Kass, ${ }^{52}$ J. Brau, ${ }^{53}$ R. Frey, ${ }^{53}$ N. B. Sinev, ${ }^{53}$ D. Strom,${ }^{53}$ E. Torrence, ${ }^{53}$ E. Feltresi,${ }^{54,54 b}$ N. Gagliardi, ${ }^{54 a, 54 b}$ M. Margoni, ${ }^{54 a, 54 b}$ M. Morandin, ${ }^{54 a}$ M. Posocco, ${ }^{54 \mathrm{a}}$ M. Rotondo, ${ }^{54 \mathrm{a}}$ G. Simi,${ }^{54 \mathrm{a}}$ F. Simonetto, ${ }^{54 \mathrm{a}, 54 \mathrm{~b}}$ R. Stroili, ${ }^{54 \mathrm{a}, 54 \mathrm{~b}}$ S. Akar, ${ }^{55}$ E. Ben-Haim, ${ }^{55}$ M. Bomben, ${ }^{55}$ G. R. Bonneaud, ${ }^{55}$ H. Briand, ${ }^{55}$ G. Calderini, ${ }^{55}$ J. Chauveau, ${ }^{55}$ O. Hamon, ${ }^{55}$ Ph. Leruste, ${ }^{55}$ G. Marchiori, ${ }^{55}$ J. Ocariz ${ }^{55}$ S. Sitt, ${ }^{55}$ M. Biasini, ${ }^{56 a, 56 b}$ E. Manoni, ${ }^{56 a, 56 b}$ S. Pacetti, ${ }^{56 a, 56 b}$ A. Rossi, ${ }^{56,56 b}$ C. Angelini, ${ }^{57 a, 57 b}$ G. Batignani, ${ }^{57 a, 57 b}$ S. Bettarini, ${ }^{57 a, 57 b}$ M. Carpinelli, ${ }^{57 a, 57 b, \pi l}$ G. Casarosa, ${ }^{57 a, 57 b}$ A. Cervelli, ${ }^{57 a, 57 b}$ F. Forti, ${ }^{57 a, 57 b}$ M. A. Giorgi, ${ }^{57 a, 57 b}$ A. Lusiani, ${ }^{57 a, 57 \mathrm{c}}$ B. Oberhof, ${ }^{57 a, 57 b}$ E. Paoloni, ${ }^{57 a, 57 b}$ A. Perez, ${ }^{57 a}$ G. Rizzo, ${ }^{57 a, 57 b}$ J. J. Walsh ${ }^{57 a}$ D. Lopes Pegna, ${ }^{58}$ J. Olsen, ${ }^{58}$ A. J. S. Smith, ${ }^{58}$ A. V. Telnov ${ }^{58}$ F. Anulli, ${ }^{59 a}$ R. Faccini,${ }^{59 a, 59 b}$ F. Ferrarotto, ${ }^{59 a}$ F. Ferroni, ${ }^{59 a, 59 b}$ M. Gaspero, ${ }^{59 a, 59 b}$ L. Li Gioi, ${ }^{59 a}$ M. A. Mazzoni, ${ }^{59 a}$ G. Piredda, ${ }^{59 a}$ C. Bünger,${ }^{60}$ O. Grünberg, ${ }^{60}$ T. Hartmann, ${ }^{60}$ T. Leddig, ${ }^{60}$ C. Voß ${ }^{60}$ R. Waldi, ${ }^{60}$ T. Adye, ${ }^{61}$ E. O. Olaiya, ${ }^{61}$ F. F. Wilson, ${ }^{61}$ S. Emery, ${ }^{62}$ G. Hamel de Monchenault,${ }^{62}$ G. Vasseur, ${ }^{62}$ Ch. Yèche, ${ }^{62}$ D. Aston, ${ }^{63}$ D. J. Bard, ${ }^{63}$ R. Bartoldus, ${ }^{63}$ J. F. Benitez, ${ }^{63}$ C. Cartaro, ${ }^{63}$ M. R. Convery, ${ }^{63}$ J. Dorfan, ${ }^{63}$ G. P. Dubois-Felsmann, ${ }^{63}$ W. Dunwoodie, ${ }^{63}$ M. Ebert, ${ }^{63}$ R. C. Field,${ }^{63}$ M. Franco Sevilla ${ }^{63}$ B. G. Fulsom, ${ }^{63}$ A. M. Gabareen, ${ }^{63}$ M. T. Graham, ${ }^{63}$ P. Grenier, ${ }^{63}$ C. Hast, ${ }^{63}$ W. R. Innes, ${ }^{63}$ M. H. Kelsey, ${ }^{63}$ P. Kim, ${ }^{63}$ M. L. Kocian, ${ }^{63}$ D. W. G. S. Leith, ${ }^{63}$ P. Lewis, ${ }^{63}$ B. Lindquist, ${ }^{63}$ S. Luitz, ${ }^{63}$ V. Luth, ${ }^{63}$ H. L. Lynch, ${ }^{63}$ D. B. MacFarlane, ${ }^{63}$ D. R. Muller,${ }^{63}$ H. Neal, ${ }^{63}$ S. Nelson, ${ }^{63}$ M. Perl, ${ }^{63}$ T. Pulliam, ${ }^{63}$ B. N. Ratcliff, ${ }^{63}$ A. Roodman ${ }^{63}$ A. A. Salnikov, ${ }^{63}$ R. H. Schindler, ${ }^{63}$ A. Snyder, ${ }^{63}$ D. Su, ${ }^{63}$ M. K. Sullivan, ${ }^{63}$ J. Va'vra, ${ }^{63}$ A. P. Wagner, ${ }^{63}$ W. J. Wisniewski, ${ }^{63}$ M. Wittgen, ${ }^{63}$ D. H. Wright, ${ }^{63}$ H. W. Wulsin, ${ }^{63}$ C. C. Young, ${ }^{63}$ V. Ziegler, ${ }^{63}$ W. Park, ${ }^{64}$ M. V. Purohit, ${ }^{64}$ R. M. White,${ }^{64}$ J. R. Wilson, ${ }^{64}$ A. Randle-Conde, ${ }^{65}$ S. J. Sekula ${ }^{65}$ M. Bellis,${ }^{66}$ P. R. Burchat ${ }^{66}$ T. S. Miyashita, ${ }^{66}$ E. M. T. Puccio, ${ }^{66}$ M. S. Alam, ${ }^{67}$ J. A. Ernst, ${ }^{67}$

R. Gorodeisky, ${ }^{68}$ N. Guttman, ${ }^{68}$ D. R. Peimer, ${ }^{68}$ A. Soffer,${ }^{68}$ P. Lund, ${ }^{69}$ S. M. Spanier, ${ }^{69}$ J. L. Ritchie, ${ }^{70}$ A. M. Ruland, ${ }^{70}$ R. F. Schwitters ${ }^{70}$ B.C. Wray ${ }^{70}$ J. M. Izen, ${ }^{71}$ X. C. Lou ${ }^{71}$ F. Bianchi, ${ }^{72 a, 72 b}$ D. Gamba, ${ }^{72 a, 72 b}$ S. Zambito, ${ }^{72 a, 72 b}$ L. Lanceri, ${ }^{73 a, 73 b}$ L. Vitale, ${ }^{73 a, 73 b}$ F. Martinez-Vidal, ${ }^{74}$ A. Oyanguren, ${ }^{74}$ P. Villanueva-Perez, ${ }^{74}$ H. Ahmed, ${ }^{75}$ J. Albert, ${ }^{75}$ Sw. Banerjee, ${ }^{75}$ F. U. Bernlochner, ${ }^{75}$ H. H. F. Choi, ${ }^{75}$ G. J. King, ${ }^{75}$ R. Kowalewski, ${ }^{75}$ M. J. Lewczuk, ${ }^{75}$ I. M. Nugent, ${ }^{75}$ J. M. Roney, ${ }^{75}$ R. J. Sobie, ${ }^{75}$ N. Tasneem,${ }^{75}$ T. J. Gershon, ${ }^{76}$ P. F. Harrison, ${ }^{76}$ T. E. Latham, ${ }^{76}$ H. R. Band, ${ }^{77}$ S. Dasu, ${ }^{77}$ Y. Pan, ${ }^{77}$ R. Prepost, ${ }^{77}$ and S. L. $\mathrm{Wu}^{77}$ 
(BABAR Collaboration)

\author{
${ }^{1}$ Laboratoire d'Annecy-le-Vieux de Physique des Particules (LAPP), Université de Savoie, \\ CNRS/IN2P3, F-74941 Annecy-Le-Vieux, France \\ ${ }^{2}$ Departament ECM, Facultat de Fisica, Universitat de Barcelona, E-08028 Barcelona, Spain \\ ${ }^{3 a}$ INFN Sezione di Bari, I-70126 Bari, Italy \\ ${ }^{3 \mathrm{~b}}$ Dipartimento di Fisica, Università di Bari, I-70126 Bari, Italy \\ ${ }^{4}$ Institute of Physics, University of Bergen, N-5007 Bergen, Norway \\ ${ }^{5}$ Lawrence Berkeley National Laboratory and University of California, Berkeley, California 94720, USA \\ ${ }^{6}$ Institut für Experimentalphysik 1, Ruhr Universität Bochum, D-44780 Bochum, Germany \\ ${ }^{7}$ University of British Columbia, Vancouver, British Columbia, Canada V6T 1 Z1 \\ ${ }^{8}$ Brunel University, Uxbridge, Middlesex UB8 3PH, United Kingdom \\ ${ }^{9}$ Budker Institute of Nuclear Physics, Novosibirsk 630090, Russia \\ ${ }^{10}$ University of California at Irvine, Irvine, California 92697, USA \\ ${ }^{11}$ University of California at Riverside, Riverside, California 92521, USA \\ ${ }^{12}$ University of California at Santa Barbara, Santa Barbara, California 93106, USA \\ ${ }^{13}$ Institute for Particle Physics, University of California at Santa Cruz, Santa Cruz, California 95064, USA \\ ${ }^{14}$ California Institute of Technology, Pasadena, California 91125, USA \\ ${ }^{15}$ University of Cincinnati, Cincinnati, Ohio 45221, USA \\ ${ }^{16}$ University of Colorado, Boulder, Colorado 80309, USA \\ ${ }^{17}$ Colorado State University, Fort Collins, Colorado 80523, USA \\ ${ }^{18}$ Fakultät Physik, Technische Universität Dortmund, D-44221 Dortmund, Germany \\ ${ }^{19}$ Institut für Kern- und Teilchenphysik, Technische Universität Dresden, D-01062 Dresden, Germany \\ ${ }^{20}$ Laboratoire Leprince-Ringuet, Ecole Polytechnique, CNRS/IN2P3, F-91128 Palaiseau, France \\ ${ }^{21}$ University of Edinburgh, Edinburgh EH9 3JZ, United Kingdom \\ ${ }^{22 \mathrm{a}}$ INFN Sezione di Ferrara, I-44100 Ferrara, Italy \\ ${ }^{22 \mathrm{~b}}$ Dipartimento di Fisica, Università di Ferrara, I-44100 Ferrara, Italy \\ ${ }^{23}$ INFN Laboratori Nazionali di Frascati, I-00044 Frascati, Italy \\ ${ }^{24 a}$ INFN Sezione di Genova, I-16146 Genova, Italy \\ ${ }^{24 \mathrm{~b}}$ Dipartimento di Fisica, Università di Genova, I-16146 Genova, Italy \\ ${ }^{25}$ Indian Institute of Technology Guwahati, Guwahati, Assam 781 039, India \\ ${ }^{26}$ Harvard University, Cambridge, Massachusetts 02138, USA \\ ${ }^{27}$ Harvey Mudd College, Claremont, California 91711, USA \\ ${ }^{28}$ Physikalisches Institut, Universität Heidelberg, Philosophenweg 12, D-69120 Heidelberg, Germany \\ ${ }^{29}$ Institut für Physik, Humboldt-Universität zu Berlin, Newtonstraße 15, D-12489 Berlin, Germany \\ ${ }^{30}$ Imperial College London, London SW7 2AZ, United Kingdom \\ ${ }^{31}$ University of Iowa, Iowa City, Iowa 52242, USA \\ ${ }^{32}$ Iowa State University, Ames, Iowa 50011-3160, USA \\ ${ }^{33}$ Johns Hopkins University, Baltimore, Maryland 21218, USA \\ ${ }^{34}$ Laboratoire de l'Accélérateur Linéaire, IN2P3/CNRS et Université Paris-Sud 11, \\ Centre Scientifique d'Orsay, B. P. 34, F-91898 Orsay Cedex, France \\ ${ }^{35}$ Lawrence Livermore National Laboratory, Livermore, California 94550, USA \\ ${ }^{36}$ University of Liverpool, Liverpool L69 7ZE, United Kingdom \\ ${ }^{37}$ Queen Mary, University of London, London E1 4NS, United Kingdom \\ ${ }^{38}$ Royal Holloway and Bedford New College, University of London, Egham, Surrey TW20 OEX, United Kingdom \\ ${ }^{39}$ University of Louisville, Louisville, Kentucky 40292, USA \\ ${ }^{40}$ Institut für Kernphysik, Johannes Gutenberg-Universität Mainz, D-55099 Mainz, Germany \\ ${ }^{41}$ University of Manchester, Manchester M13 9PL, United Kingdom \\ ${ }^{42}$ University of Maryland, College Park, Maryland 20742, USA \\ ${ }^{43}$ University of Massachusetts, Amherst, Massachusetts 01003, USA \\ ${ }^{44}$ Laboratory for Nuclear Science, Massachusetts Institute of Technology, Cambridge, Massachusetts 02139, USA \\ ${ }^{45}$ Mc Gill University, Montréal, Québec, Canada H3A $2 T 8$ \\ ${ }^{46 a}$ INFN Sezione di Milano, I-20133 Milano, Italy \\ ${ }^{46 \mathrm{~b}}$ Dipartimento di Fisica, Università di Milano, I-20133 Milano, Italy \\ ${ }^{47}$ University of Mississippi, University, Mississippi 38677, USA \\ ${ }^{48}$ Physique des Particules, Université de Montréal, Montréal, Québec, Canada H3C 3J7 \\ ${ }^{49 a}$ INFN Sezione di Napoli, I-80126 Napoli, Italy \\ ${ }^{49 \mathrm{~b}}$ Dipartimento di Scienze Fisiche, Università di Napoli Federico II, I-80126 Napoli, Italy \\ ${ }^{50}$ NIKHEF, National Institute for Nuclear Physics and High Energy Physics, NL-1009 DB Amsterdam, The Netherlands
}


${ }^{51}$ University of Notre Dame, Notre Dame, Indiana 46556, USA

${ }^{52}$ Ohio State University, Columbus, Ohio 43210, USA

${ }^{53}$ University of Oregon, Eugene, Oregon 97403, USA

${ }^{54 a}$ INFN Sezione di Padova, I-35131 Padova, Italy

${ }^{54 b}$ Dipartimento di Fisica, Università di Padova, I-35131 Padova, Italy

${ }^{55}$ Laboratoire de Physique Nucléaire et de Hautes Energies, IN2P3/CNRS, Université Pierre et Marie Curie-Paris6,

Université Denis Diderot-Paris7, F-75252 Paris, France

${ }^{56}$ INFN Sezione di Perugia, I-06100 Perugia, Italy

${ }^{56 \mathrm{~b}}$ Dipartimento di Fisica, Università di Perugia, I-06100 Perugia, Italy

${ }^{57}$ INFN Sezione di Pisa, I-56127 Pisa, Italy

${ }^{57 \mathrm{~b}}$ Dipartimento di Fisica, Università di Pisa, I-56127 Pisa, Italy

${ }^{57 \mathrm{c}}$ Scuola Normale Superiore di Pisa, I-56127 Pisa, Italy

${ }^{58}$ Princeton University, Princeton, New Jersey 08544, USA

${ }^{59 a}$ INFN Sezione di Roma, I-00185 Roma, Italy

${ }^{59 \mathrm{~b}}$ Dipartimento di Fisica, Università di Roma La Sapienza, I-00185 Roma, Italy

${ }^{60}$ Universität Rostock, D-18051 Rostock, Germany

${ }^{61}$ Rutherford Appleton Laboratory, Chilton, Didcot, Oxon OX11 OQX, United Kingdom

${ }^{62}$ CEA, Irfu, SPP, Centre de Saclay, F-91191 Gif-sur-Yvette, France

${ }^{63}$ SLAC National Accelerator Laboratory, Stanford, California 94309 USA

${ }^{64}$ University of South Carolina, Columbia, South Carolina 29208, USA

${ }^{65}$ Southern Methodist University, Dallas, Texas 75275, USA

${ }^{66}$ Stanford University, Stanford, California 94305-4060, USA

${ }^{67}$ State University of New York, Albany, New York 12222, USA

${ }^{68}$ Tel Aviv University, School of Physics and Astronomy, Tel Aviv 69978, Israel

${ }^{69}$ University of Tennessee, Knoxville, Tennessee 37996, USA

${ }^{70}$ University of Texas at Austin, Austin, Texas 78712, USA

${ }^{71}$ University of Texas at Dallas, Richardson, Texas 75083, USA

${ }^{72 \mathrm{a}}$ INFN Sezione di Torino, I-10125 Torino, Italy

${ }^{72 b}$ Dipartimento di Fisica Sperimentale, Università di Torino, I-10125 Torino, Italy

${ }^{73 a}$ INFN Sezione di Trieste, I-34127 Trieste, Italy

${ }^{73 b}$ Dipartimento di Fisica, Università di Trieste, I-34127 Trieste, Italy

${ }^{74}$ IFIC, Universitat de Valencia-CSIC, E-46071 Valencia, Spain

${ }^{75}$ University of Victoria, Victoria, British Columbia, Canada V8W $3 P 6$

${ }^{76}$ Department of Physics, University of Warwick, Coventry CV4 7AL, United Kingdom

${ }^{77}$ University of Wisconsin, Madison, Wisconsin 53706, USA

(Received 23 October 2012; published 10 October 2013)

We search for a low-mass scalar $C P$-odd Higgs boson, $A^{0}$, produced in the radiative decay of the upsilon resonance and decaying into a $\tau^{+} \tau^{-}$pair: $\Upsilon(1 S) \rightarrow \gamma A^{0}$. The production of $\Upsilon(1 S)$ mesons is tagged by $\Upsilon(2 S) \rightarrow \pi^{+} \pi^{-} \Upsilon(1 S)$ transitions, using a sample of $(98.3 \pm 0.9) \times 10^{6} \Upsilon(2 S)$ mesons collected by the BABAR detector. We find no evidence for a Higgs boson in the mass range $3.5 \leq m_{A^{0}} \leq$ 9.2 $\mathrm{GeV}$, and combine these results with our previous search for the tau decays of the light Higgs in radiative $Y(3 S)$ decays, setting limits on the coupling of $A^{0}$ to the $b \bar{b}$ quarks in the range 0.09-1.9. Our measurements improve the constraints on the parameters of the next-to-minimal-supersymmetric Standard Model and similar theories with low-mass scalar degrees of freedom.

\footnotetext{
*Deceased.

${ }^{\dagger}$ Present address: University of Tabuk, Tabuk 71491, Saudi Arabia.

${ }^{\ddagger}$ Also at Università di Perugia, Dipartimento di Fisica, Perugia, Italy.

${ }^{\S}$ Present address: University of Huddersfield, Huddersfield HD1 3DH, United Kingdom.

"Present address: University of South Alabama, Mobile, Alabama 36688, USA.

${ }_{\text {II }}$ Also at Università di Sassari, Sassari, Italy.
}

The Higgs boson is a scalar elementary particle predicted by the Higgs mechanism [1] which attempts to explain the origin of mass of the elementary particles within the Standard Model (SM) [2]. Present experimental evidence suggests a Higgs-like state with the mass of $\approx 126 \mathrm{GeV}$ [3]. However, low-mass Higgs states with masses of $\mathcal{O}(10 \mathrm{GeV})$ appear in several extensions to the SM [4], such as the next-to-minimal supersymmetric Standard Model (NMSSM) [5]. The NMSSM adds a singlet superfield to the minimal supersymmetric Standard Model, solving the so-called naturalness problem. The NMSSM 
contains two charged Higgs bosons, three neutral $C P$-even bosons, and two $C P$-odd bosons. The lightest $C P$-odd state, $A^{0}$, could have a mass below the $b \bar{b}$ production threshold, avoiding detection at LEP [4]. Such a particle could be produced in radiative $Y \rightarrow \gamma A^{0}$ decays [6] with a branching fraction as large as $10^{-4}$ for the narrow states $\Upsilon(n S)$ (where $n \leq 3$ ), depending on the $A^{0}$ mass and couplings [5], making it accessible at B-factories. Thus, constraining the low-mass NMSSM Higgs sector is important for understanding the recent LHC discovery [7].

Searches for $A^{0}$ decays into $\mu^{+} \mu^{-}$[8], $\tau^{+} \tau^{-}$[9], invisible [10], and hadronic [11] final states have been performed by $B A B A R$, so far with null results. In particular, limits on the product of branching fractions $\mathcal{B}\left(\Upsilon(3 S) \rightarrow \gamma A^{0}\right) \times$ $\mathcal{B}\left(A^{0} \rightarrow \tau^{+} \tau^{-}\right)$have been set at $(1.5-16) \times 10^{-5}$ in the mass range $4.03<m_{A^{0}}<10.10 \mathrm{GeV}$ [9]. The CLEO Collaboration has set limits on the branching ratio product $\mathcal{B}\left(Y(1 S) \rightarrow \gamma A^{0}\right) \times \mathcal{B}\left(A^{0} \rightarrow \tau^{+} \tau^{-}\right)$in the range $10^{-5}-10^{-4}$ for masses $m_{A^{0}}<9.2 \mathrm{GeV}$ [12].

This paper describes a search for decays of the $Y(1 S)$ resonance into a photon and a light scalar $C P$-odd Higgs boson $A^{0}$, which then decays into a pair of tau leptons. The $Y(1 S)$ resonance is produced from the $Y(2 S)$ resonance with the emission of two charged pions. The reaction chain is $e^{+} e^{-} \rightarrow \Upsilon(2 S) \rightarrow \pi^{+} \pi^{-} \Upsilon(1 S), Y(1 S) \rightarrow \gamma A^{0}$, $A^{0} \rightarrow \tau^{+} \tau^{-}$. We identify the $\Upsilon(1 S)$ by the dipion transition; the production and decay of the Higgs candidates are identified by the photon and the two charged tracks from one-prong decays of the two tau leptons.

This analysis is based on a sample of $(98.3 \pm 0.9) \times 10^{6}$ $Y(2 S)$ decays collected at the $Y(2 S)$ resonance with the $B A B A R$ detector [13] at the PEP-II asymmetric-energy $e^{+} e^{-}$collider at the SLAC National Accelerator Laboratory. This sample corresponds to an integrated luminosity of $14 \mathrm{fb}^{-1}$. We also use a sample of $28 \mathrm{fb}^{-1}$ taken at the $Y(3 S)$ resonance for studies of the QED (continuum) backgrounds and the optimization of the selection of the $\Upsilon(2 S)$ dipion transition candidates. $Y(3 S)$ decays are rejected by the analysis selection criteria due to their different kinematics distributions compared with the $Y(2 S)$ decays, and therefore the $Y(3 S)$ events form a pure high-statistics continuum QED sample. An additional data set of $1.4 \mathrm{fb}^{-1}$ taken at a center-of-mass (CM) energy $30 \mathrm{MeV}$ below the $\Upsilon(2 S)$ mass is used for studies of systematic effects. We use Monte Carlo (MC) simulated samples of signal and $Y(1 S)$ background events $[14,15]$ to tune the selection of the Higgs events. The tau-lepton branching fractions are fixed to the values of Ref. [16]. The BABAR detector, including the instrumented flux return, the electromagnetic calorimeter, and the tracking and particle identification (PID) systems, is described in detail elsewhere [13,17].

A signal candidate consists of a photon plus four charged tracks: $\pi^{+} \pi^{-}$from the $\Upsilon(2 S) \rightarrow \pi^{+} \pi^{-} \Upsilon(1 S)$ transition, and the one-prong decays of the two tau leptons. The event may contain as many as 19 additional photons with laboratory energy greater than $30 \mathrm{MeV}$, mostly from beam-induced backgrounds, but no additional charged tracks. Additional signal candidates may be formed using these extra photons, but a single final candidate per event is selected, as described below.

We select events where at least one tau lepton decays leptonically, resulting in five different combinations of tau lepton daughters: $e e, e \mu, e \pi, \mu \mu, \mu \pi$. Events in which both tau leptons decay hadronically suffer from significantly larger and poorly modeled backgrounds than the leptonic channels, and are therefore excluded. The tau lepton daughters are identified using multivariate discriminants based on the information from all subdetectors. Typical PID efficiencies are 98\% (e), 90\% $(\mu)$, and $97 \%$ $(\pi)$, while the typical pion misidentification rates are less than $0.5 \%(e)$ and $5 \%(\mu)$. Requirements on the electromagnetic shower shapes of the primary photon candidates are also imposed to improve the signal purity, and events with $\pi^{0}$ candidates, formed from pairs of photons with invariant mass satisfying $100<m_{\gamma \gamma}<160 \mathrm{MeV}$ and laboratory energy above $200 \mathrm{MeV}$, are discarded.

In order to achieve a balanced selection efficiency that is as uniform as a function of the reconstructed Higgs mass $m_{A^{0}}$ (or photon energy) as possible, we optimize the selection in two Higgs mass intervals: $3.6 \leq m_{A^{0}} \leq 8.0 \mathrm{GeV}$ ( $\mathrm{L}$ range) and $8.0<m_{A^{0}} \leq 9.2 \mathrm{GeV}$ (H range). The choice of the mass ranges is motivated by the rapidly varying signal-to-background ratio at low photon energies (which correspond to higher $m_{A^{0}}$ for the signal), and differences in kinematics for each photon energy range.

The masses of the $Y(1 S)$ and $A^{0}$ candidates are calculated from two primary kinematic variables:

$$
\begin{gathered}
m_{\text {recoil }}^{2}=M_{\Upsilon(2 S)}^{2}+m_{\pi \pi}^{2}-2 M_{\Upsilon(2 S)} E_{\pi \pi}^{C M}, \\
m_{X}^{2}=\left(P_{e^{+} e^{-}}-P_{\pi \pi}-P_{\gamma}\right)^{2} .
\end{gathered}
$$

Here $m_{\text {recoil }}$ is the recoil mass of the dipion system [which peaks at the value of the $Y(1 S)$ mass for signal], $m_{X}^{2}$ is the mass recoiling against the signal photon in the $Y(1 S)$ frame (which peaks near the square of the expected Higgs mass, $m_{A^{0}}^{2}$, and is linear in the photon energy), and $P$ denotes the four-momentum.

In order to reject backgrounds, we train two multilayer perceptron neural networks (NN) [18]: a pion discriminant $\left(\mathcal{N}_{\pi}\right)$, which describes the kinematics of the process $\Upsilon(2 S) \rightarrow \pi^{+} \pi^{-} \Upsilon(1 S)$, and a tau discriminant $\left(\mathcal{N}_{\tau}\right)$ describing the transition $Y(1 S) \rightarrow \gamma A^{0}, A^{0} \rightarrow \tau^{+} \tau^{-}$. Each NN uses kinematic variables only weakly correlated to $m_{X}^{2}$ or $m_{\text {recoil }}$. The two discriminants are uncorrelated. The pion discriminant $\mathcal{N}{ }_{\pi}$ combines nine kinematic variables specific to the dipion system [10,19]. The discriminant $\mathcal{N}_{\tau}$ is a combination of 14 variables: the missing energy and the cosine of the polar angle of the missing momentum in the event; the extra calorimeter energy in the lab frame and the energy of the second most energetic photon in the CM 
frame; the net transverse momentum of the reconstructed signal candidate particles; the acoplanarity of the photon relative to the plane formed by the two tau decay prongs; the momentum and polar angle in the CM frame of the most energetic tau decay prong; the invariant mass, vertex probability, and the distance between the vertex and the $e^{+} e^{-}$interaction region of the two tau decay prongs; the angle between the signal photon and the most energetic tau decay prong in the event. The discriminants are trained using signal MC events $\mathrm{Y}(1 S) \rightarrow \gamma A^{0}$ in the range $4.0 \leq m_{A^{0}} \leq 9.2 \mathrm{GeV}$. The background samples for training are taken from the continuum sample for the pion discriminant and from the simulated generic $Y(1 S)$ decays for the tau discriminant.

Each $\mathrm{NN}$ outputs a value $\mathcal{N}$ close to +1 for signal and to -1 for background. Based on the NN outputs, the selection criteria for $\mathcal{N}_{\pi}$ and $\mathcal{N}_{\tau}$ are chosen to optimize $\varepsilon /(1.5+\sqrt{B})$ [20], where $\varepsilon$ is the signal efficiency, and $B$ is the expected background yield. We accept signal candidates if $\mathcal{N}_{\tau}$ is above a threshold value chosen individually for each final state and mass range. The thresholds for $\mathcal{N}_{\pi}$ are the same for all final states, but different for the two mass ranges. The typical signal efficiency and background rejection estimated from the corresponding MC samples are listed in Table I.

Due to reconstruction ambiguities, in particular extra photon candidates in the event and a large $\mu$-as- $\pi$ misidentification rate, a fraction of signal and background events have more than a single reconstructed candidate. The multiplicity of candidates per event is on average 1.8 for the simulated signal samples, 1.6 for the generic $Y(2 S)$ decays, 1.3 for the continuum sample, and 1.5 for the data. We select a single candidate based on (1) the highest value of $\mathcal{N}_{\pi}$, then, if multiple candidates still remain, (2) the highest value of $\mathcal{N}_{\tau}$, and finally, (3) the tau decay final state with the highest signal/background ratio.

We further suppress the continuum background by applying a cut on the mass recoiling against the dipion system $m_{\text {recoil }}$ :

$$
\left|m_{\text {recoil }}-\left\langle m_{\text {recoil }}\right\rangle\right|<10 \mathrm{MeV},
$$

TABLE I. Typical selection efficiencies (SE) and background rejection factors (BR) for the two neural network discriminants $\mathcal{N}_{\pi}$ and $\mathcal{N}_{\tau}$ in the $\mathrm{L}\left(3.6 \leq m_{A^{0}} \leq 8.0 \mathrm{GeV}\right)$ and $\mathrm{H}(8.0<$ $\left.m_{A^{0}} \leq 9.2 \mathrm{GeV}\right)$ ranges. The $\mathrm{SE}$ and $\mathrm{BR}$ factors are relative to the preselection that requires four tracks and a real photon in the final state, and are averaged over each mass range.

\begin{tabular}{lccc}
\hline \hline Mass range & Discriminant & SE (\%) & BR (\%) \\
\hline $\mathrm{L}$ & $\mathcal{N}_{\pi}$ & 76 & 99 \\
$\mathrm{H}$ & $\mathcal{N}_{\pi}^{\pi}$ & 72 & 99 \\
$\mathrm{~L}$ & $\mathcal{N}_{\tau}$ & 80 & 97 \\
$\mathrm{H}$ & $\mathcal{N}_{\tau}$ & 30 & 99 \\
\hline \hline
\end{tabular}

where $\left\langle m_{\text {recoil }}\right\rangle$ is the expected location of the $Y(1 S)$ peak, determined by the mass difference between the $Y(2 S)$ and $\Upsilon(1 S)$ mesons [21]. The final signal selection efficiency varies between $1 \%$ and $4.5 \%$ (Fig. 1), and is lowest at the highest masses (lowest photon energy).

We extract the yield of signal events as a function of $m_{A^{0}}$ in the interval $3.6 \leq m_{A^{0}} \leq 9.2 \mathrm{GeV}$ by performing a series of maximum likelihood fits in steps of $m_{A^{0}}$. We perform one-dimensional unbinned extended maximum likelihood (ML) fits to the distribution of $m_{X}^{2}$ in the intervals $12 \leq m_{X}^{2} \leq 72 \mathrm{GeV}^{2}$ ( $\mathrm{L}$ range) and $49 \leq m_{X}^{2} \leq$ $89 \mathrm{GeV}^{2}$ (H range). The fit intervals overlap to provide sufficient sidebands for all values of $m_{A^{0}}$. The likelihood contains contributions from signal, which is expected to peak near the Higgs mass squared, and from the smooth background function, arising from continuum and radiative leptonic $Y(1 S)$ backgrounds. We search for the $A^{0}$ in varying mass steps that correspond to approximately half of the expected resolution on $m_{A^{0}}$, as described below. A total of 201 mass points are sampled.

We use signal MC samples $\Upsilon(2 S) \rightarrow \pi^{+} \pi^{-} \Upsilon(1 S)$, $\Upsilon(1 S) \rightarrow \gamma A^{0}$ generated at nine (seven) different values of $m_{A^{0}}$ in the $\mathrm{L}(\mathrm{H})$ mass range to determine the signal probability density functions (PDFs) in $m_{X}^{2}$ and selection efficiencies. We interpolate these distributions and efficiencies between fixed $m_{A^{0}}$ points, correcting for known small differences between data and MC simulations. The signal PDFs only include events in which the simulated signal photon, $\pi^{+} \pi^{-}$tracks, and the tracks from the decay of the tau leptons are correctly reconstructed. The signal efficiency, however, includes contributions from events in which one of the final-state particles may be misreconstructed. The signal PDF is modeled as a Crystal Ball function [22]. We parametrize the background PDFs as

$$
\begin{aligned}
& f(z)=\left(\operatorname{Erf}\left[\alpha\left(z-z_{\min }\right)\right]+1\right) C_{3}(z) \quad(\mathrm{L} \text { range }) ; \\
& f(z)=\left(1-\frac{z}{z_{\max }}\right)^{\beta} \exp \left[C_{2}(z)\right] \quad(\text { H range }),
\end{aligned}
$$

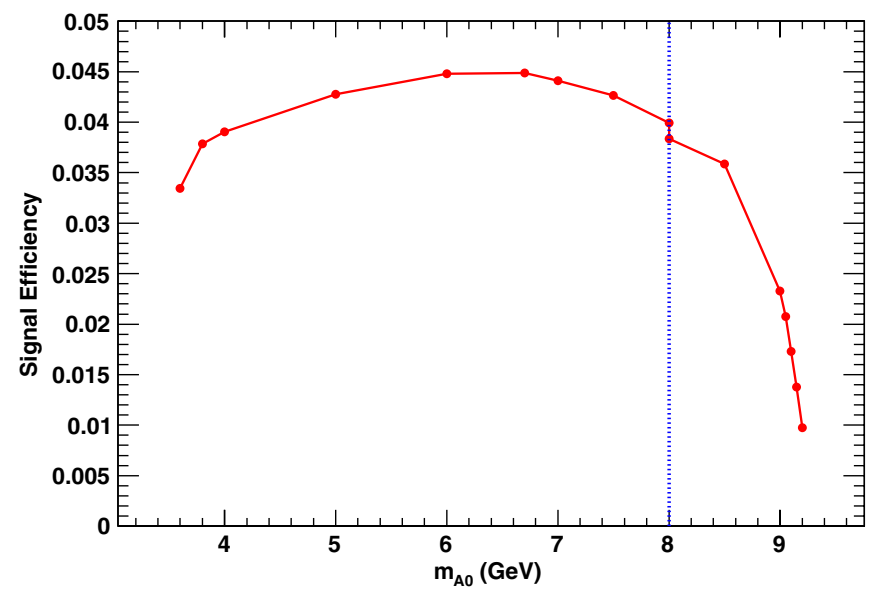

FIG. 1 (color online). Signal efficiency as a function of $m_{A^{0}}$ in the $\mathrm{L}$ and $\mathrm{H}$ mass ranges (separated by the vertical blue line). 
where $z \equiv m_{X}^{2}, C_{n}(z)$ is an $n$ th-order Chebyshev polynomial (with different parameters for each mass range), $\alpha$ and $\beta$ are threshold parameters, and $z_{\min }=12.5 \mathrm{GeV}^{2}$ and $z_{\max }=88.9 \mathrm{GeV}^{2}$ are determined by the kinematic end points of the photon energy spectrum in the luminosity-weighted mixture of simulated generic $Y(1 S)$ decays and continuum sample. A common background PDF describes all five decay channels adequately well. Parameters of the Chebyshev polynomials $C_{n}(z)$ and the threshold parameters $\alpha$ and $\beta$ are determined from a fit to the data distribution of $m_{X}^{2}$ with the signal yield fixed to zero. This accounts for uncertainties in the modeling of the radiative $Y(1 S)$ decays and additional backgrounds that may not be well described by the continuum sample, such as two-photon events with low photon energy (high $m_{X}^{2}$ ).

For each $m_{A^{0}}$ hypothesis, we determine two parameters: the background yield $N_{\text {bkg }}$ and the signal yield $N_{\text {sig. In the } \mathrm{H}}$ range, we also allow the two coefficients of $C_{2}(z)$ and the parameter $\beta$ to vary in the fit. The fit is performed simultaneously over the distributions in each $\tau^{+} \tau^{-}$decay channel, taking advantage of the difference in the signal-to-background ratios over the decay channels. The fraction of events in each channel is fixed from MC samples for signal, and from the luminosity-weighted mixture of simulated generic $Y(1 S)$ decays and continuum sample for the background.

Each fixed nuisance parameter in the fit is varied according to its uncertainty; correlations between parameters are taken into account. The systematic uncertainties for this measurement can be divided into two categories:

(i) Additive errors: uncertainties on the event yield, which do not scale with the number of reconstructed signal events. These include uncertainties of the parameters fixed in the fit (PDF shape parameters for signal and backgrounds) and a small bias in the ML fit. These uncertainties reduce the significance of any observed signal [23].

(ii) Multiplicative errors: uncertainties that scale with the number of reconstructed signal events. These include uncertainties on the reconstruction efficiency, the ML fit bias which scales with the true number of signal events, the uncertainty in the number of produced $Y(2 S)$ mesons, and the uncertainty in the branching fraction of $\Upsilon(2 S) \rightarrow \pi^{+} \pi^{-} \Upsilon(1 S)$.

We compute the average bias of the ML fit for a set of generated $N_{\text {sig }}$ values using a large ensemble of simulated pseudoexperiments. In each pseudoexperiment, the signal events are fully simulated, and the background events are sampled from their PDFs. We determine the fit bias that is independent of $N_{\text {sig }}$ and is part of the additive uncertainties, as well as the bias which scales linearly with $N_{\text {sig }}$, and can be thought of as a "fit inefficiency," i.e. a relative correction to the signal reconstruction efficiency. The bias arises from imperfections in modeling of the signal PDFs, from events in which signal candidates are misreconstructed, and from low-statistics properties of the ML estimators.
We see that a correction of $3.1 \pm 1.1 \%$ ( $\mathrm{L}$ range) and $7.6 \pm$ $1.4 \%$ ( $\mathrm{H}$ range) has to be applied. The additive parts of the fit bias are small.

The signal efficiencies determined in MC simulations are corrected by several multiplicative effects:

(i) Tracking and dipion selection efficiency.-These corrections and their uncertainties have been determined [10] using a clean sample of four-track final states from decays $\Upsilon(2 S) \rightarrow \pi^{+} \pi^{-} \Upsilon(1 S), \Upsilon(1 S) \rightarrow$ $\mu^{+} \mu^{-}$. The data/MC ratio of $0.97 \pm 0.02$ includes the uncertainties due to the number of produced $\Upsilon(2 S)$ events, dipion branching ratio $Y(2 S) \rightarrow$ $\pi^{+} \pi^{-} Y(1 S)$, dipion reconstruction efficiency, the efficiency of reconstructing two additional (energetic) charged tracks, trigger uncertainties, and the selection efficiency for the pion discriminant $\mathcal{N}_{\pi}$. The uncertainty is dominated by the error on $Y(1 S) \rightarrow \mu^{+} \mu^{-}$branching ratio [16].

(ii) Photon selection efficiency. $-\mathrm{A}$ correction of $0.967 \pm 0.017$ is determined from a high-statistics $e^{+} e^{-} \rightarrow \gamma \gamma$ sample in which one of the photons converts in the inner detector material to produce a detectable $e^{+} e^{-}$pair [10].

(iii) Neural network selection efficiency.-We evaluate the systematic uncertainty due to possible data/MC differences in the distributions of the NN discriminant $\mathcal{N}_{\tau}$ using an inclusive background sample. We select signal-like events that pass the requirement $\mathcal{N}_{\tau}>0$ and compute the ratio of partial selection efficiencies for the actual $\mathcal{N}_{\tau}$ thresholds for the data and the background. These ratios are $1.038 \pm$ 0.013 ( $\mathrm{L}$ range) and $0.991 \pm 0.014$ ( $\mathrm{H}$ range)

The total correction to the efficiency is a product of all corrections discussed above:

$$
\begin{array}{ll}
\varepsilon_{\mathrm{data}} / \varepsilon_{\mathrm{MC}}=0.943 \pm 0.031 & (\text { L range }) \\
\varepsilon_{\mathrm{data}} / \varepsilon_{\mathrm{MC}}=0.859 \pm 0.033 & (\text { H range })
\end{array}
$$

We compute the statistical significance of a particular fit centered at $m_{A^{0}}$ as $\mathcal{S}=\sqrt{2 \log \left(\mathcal{L}_{\max } / \mathcal{L}_{0}\right)}$, where $\mathcal{L}_{\max }$ is the maximum likelihood value for a fit with a free signal yield, and $\mathcal{L}_{0}$ is the value of the likelihood for $N_{\text {sig }}=0$. Including additive systematic uncertainties, the most significant upward fluctuations occur at $m_{A^{0}}=6.36 \mathrm{GeV}$ with $\mathcal{S}=2.7 \sigma$ [Fig. 2(a)] and $m_{A^{0}}=8.93 \mathrm{GeV}$ with $\mathcal{S}=3.0 \sigma$ [Fig. 2(b)]. Fluctuations of $+3 \sigma$ or higher occur in $7.5 \%$ of pseudoexperiments that simulate a scan of 201 mass points with an average correlation of $94.5 \%$ between adjacent points, as observed in our data set. Therefore, we conclude that no significant $A^{0}$ signal is found.

Since we do not observe a significant excess of events above the background, we set $90 \%$ confidence level (C.L.) Bayesian upper limits on the product $\mathcal{B}\left(\Upsilon(1 S) \rightarrow \gamma A^{0}\right) \times$ $\mathcal{B}\left(A^{0} \rightarrow \tau^{+} \tau^{-}\right)$, computed with a uniform prior for $N_{\text {sig }}>0$. The limits are shown in Fig. 3. Systematic uncertainties on 
SEARCH FOR A LOW-MASS SCALAR HIGGS BOSON ...

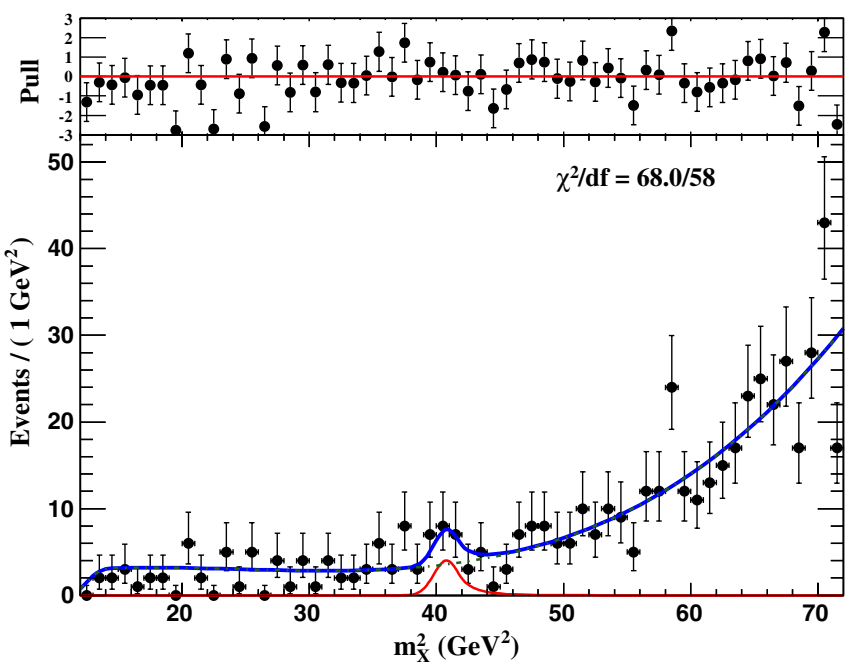

(a)

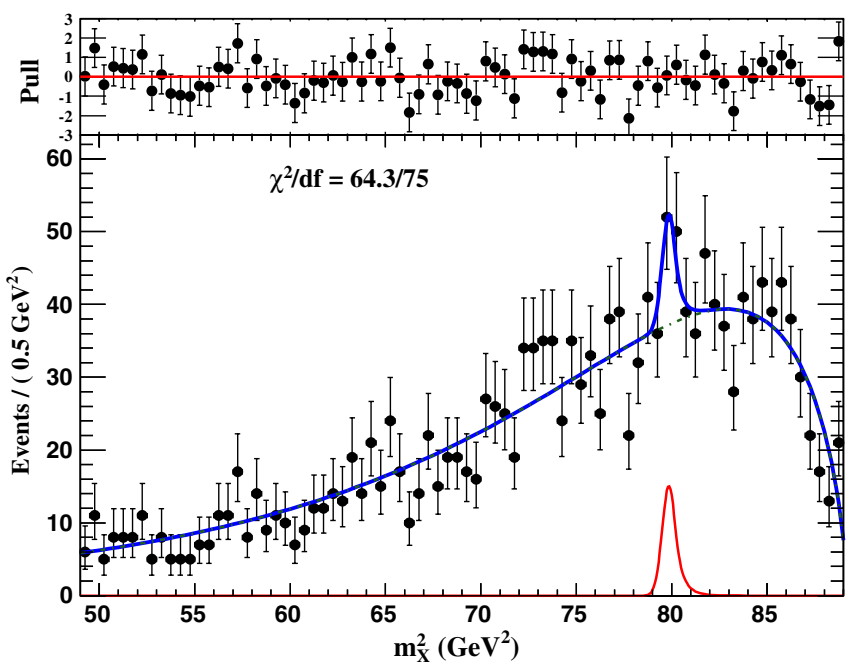

(b)

FIG. 2 (color online). Fits to the $m_{X}^{2}$ distributions in (a) $\mathrm{L}$ and (b) $\mathrm{H}$ ranges for the two particular $m_{A^{0}}$ points that return the largest upward fluctuations: (a) $m_{A^{0}}=6.36 \mathrm{GeV}$ and (b) $m_{A^{0}}=8.93 \mathrm{GeV}$. The red solid line shows the signal PDF, the green dot-dashed line is the background contribution, and the blue solid line shows the total PDF. The top plots show the fit residuals normalized by the error (pulls). The signal peaks corresponds to a statistical significance of (a) $2.7 \sigma$ and (b) $3.0 \sigma$.

$N_{\text {sig }}$ and $\varepsilon_{\text {data }}$ are included assuming their likelihood profiles are Gaussian [23].

We combine our results with the previous limits on the branching ratios $\mathcal{B}\left(\Upsilon(3 S) \rightarrow \gamma A^{0}\right) \times \mathcal{B}\left(A^{0} \rightarrow \tau^{+} \tau^{-}\right)$[9] to set a limit on the Yukawa coupling $g_{b}^{2}$ of the $b$-quark to the $A^{0}$. The branching fractions $\mathcal{B}\left(\Upsilon(n S) \rightarrow \gamma A^{0}\right)$ are related to $g_{b}$ through $[6,24,25]$

$$
\frac{\mathcal{B}\left(\Upsilon(n S) \rightarrow \gamma A^{0}\right)}{\mathcal{B}\left(\Upsilon(n S) \rightarrow l^{+} l^{-}\right)}=\frac{g_{b}^{2} G_{F} m_{b}^{2}}{\sqrt{2} \pi \alpha} \mathcal{F}_{\mathrm{QCD}}\left(1-\frac{m_{A^{0}}^{2}}{m_{\Upsilon(n S)}^{2}}\right)
$$

where $l \equiv e$ or $\mu, \alpha$ is the fine structure constant computed at the scale $m_{\Upsilon(n S)}, G_{F}$ is the Fermi constant, and $\mathcal{F}_{\mathrm{QCD}}$
PHYSICAL REVIEW D 88, 071102(R) (2013)

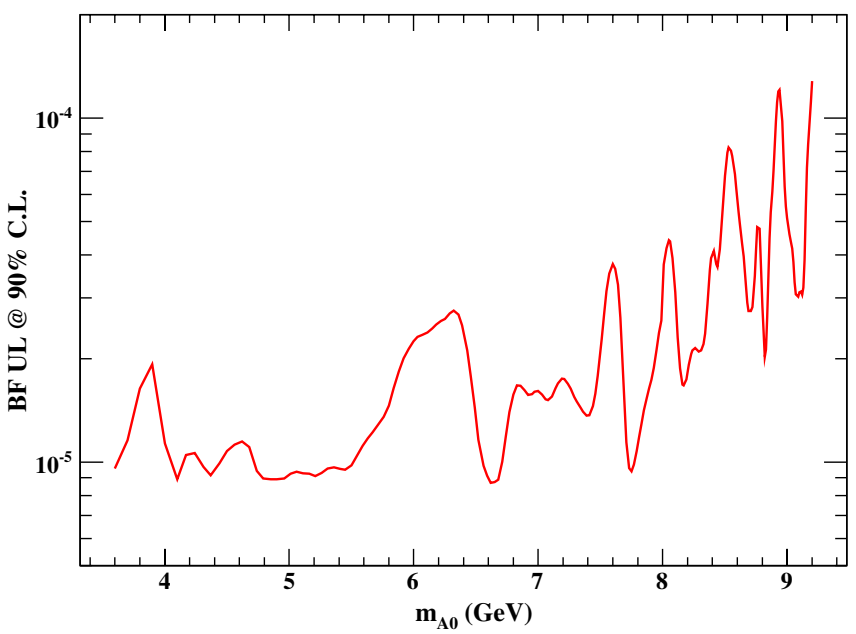

FIG. 3 (color online). $90 \%$ C.L. upper limits for $\mathcal{B}(Y(1 S) \rightarrow$ $\left.\gamma A^{0}\right) \times \mathcal{B}\left(A^{0} \rightarrow \tau^{+} \tau^{-}\right)$.

includes the $m_{A^{0}}$-dependent QCD and relativistic corrections to $\mathcal{B}\left(Y(n S) \rightarrow \gamma A^{0}\right)$ [25] and the leptonic width of $\Upsilon(n S)$ [26]. To first order in $\alpha_{S}$, the corrections range from $0 \%$ to $30 \%$ [25] and may have $\mathcal{O}(10 \%-20 \%)$ uncertainties [27]. Since the size of these uncertainties is not well estimated but is small compared to the statistical errors on $\mathcal{B}\left(Y(n S) \rightarrow \gamma A^{0}\right)$, we do not include them in the calculation of the limits on $g_{b}$ below.

We combine our data on $Y(1 S) \rightarrow \gamma A^{0}$ with the BABAR results of Ref. [9] using the full likelihood functions for $g_{b}^{2}$ at each $m_{A^{0}}$ point from this analysis, and a Gaussian approximation for the $g_{b}^{2}$ likelihood from Ref. [9]. The combined upper limits on the product $g_{b}^{2} \times \mathcal{B}\left(A^{0} \rightarrow \tau^{+} \tau^{-}\right)$ as a function of $m_{A^{0}}$ are shown in Fig. 4. They rule out

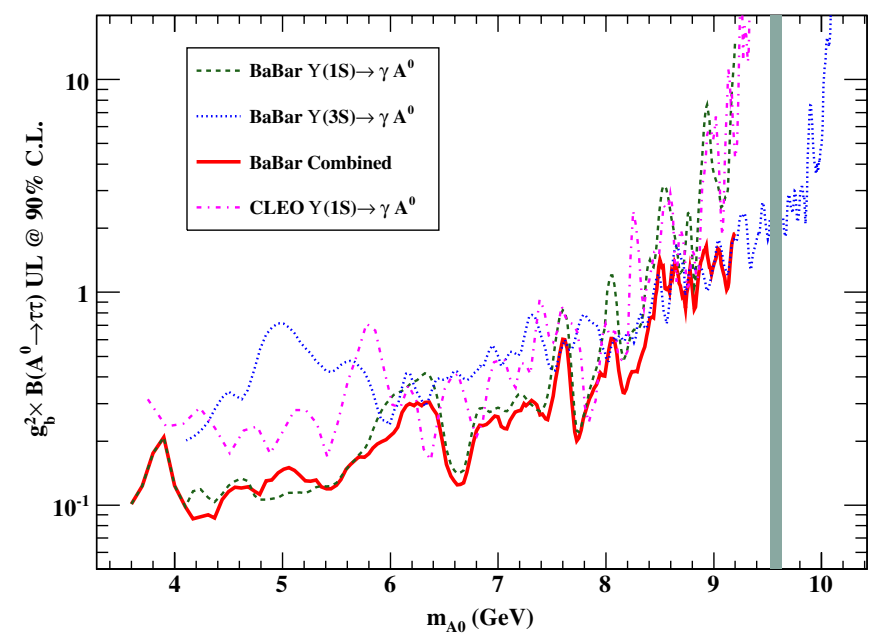

FIG. 4 (color online). $90 \%$ C.L. upper limits for Yukawa coupling $g_{b}^{2} \times \mathcal{B}\left(A^{0} \rightarrow \tau^{+} \tau^{-}\right)$. Shown are combined $B A B A R$ results (red solid line), results from this analysis only (dashed green line), the previous $B A B A R$ measurement [9] (dotted blue line), and results from the CLEO experiment [12] (dot-dashed black line). The shaded vertical bar shows the region around $\chi_{b}(2 P)$ mass excluded from Ref. [9]. 


\section{J. P. LEES et al.}

much of the parameter space preferred by NMSSM $g_{b}=$ $\tan \beta \cos \theta_{A}>1$, where $\tan \beta$ is the ratio of the Higgs vacuum expectation values and $\cos \theta_{A}$ is the fraction of the nonsinglet component in the $C P$-odd $A^{0}$ [5].

In summary, we find no evidence for the radiative decays $\Upsilon(1 S) \rightarrow \gamma A^{0}$ in which $A^{0}$ decays into a pair of tau leptons, and we set $90 \%$ C.L. upper limits on the product of branching fractions $\mathcal{B}\left(\Upsilon(1 S) \rightarrow \gamma A^{0}\right) \times \mathcal{B}\left(A^{0} \rightarrow \tau^{+} \tau^{-}\right)$ in the range $(0.9-13) \times 10^{-5}$ for $3.6 \leq m_{A^{0}} \leq 9.2 \mathrm{GeV}$. We also set $90 \%$ C.L. upper limits on the product $g_{b}^{2} \times$ $\mathcal{B}\left(A^{0} \rightarrow \tau^{+} \tau^{-}\right)$in the range $0.09-1.9$ for $m_{A^{0}} \leq 9.2 \mathrm{GeV}$. Our limits place significant constraints on NMSSM parameter space.
PHYSICAL REVIEW D 88, 071102(R) (2013)

We are grateful for the excellent luminosity and machine conditions provided by our PEP-II colleagues, and for the substantial dedicated effort from the computing organizations that support $B A B A R$. The collaborating institutions wish to thank SLAC for its support and kind hospitality. This work is supported by DOE and NSF (USA), NSERC (Canada), CEA and CNRS-IN2P3 (France), BMBF and DFG (Germany), INFN (Italy), FOM (The Netherlands), NFR (Norway), MES (Russia), MICIIN (Spain), and STFC (United Kingdom). Individuals have received support from the Marie Curie EIF (European Union), the A. P. Sloan Foundation (USA) and the Binational Science Foundation (USA-Israel).
[1] P. W. Higgs, Phys. Rev. Lett. 13, 508 (1964).

[2] S. Weinberg, Phys. Rev. Lett. 19, 1264 (1967); A. Salam, in Elementary Particle Theory, edited by N. Svartholm (Almquist and Wiksells, Stockholm, 1969), p. 367; S. L. Glashow, J. Iliopoulos, and L. Maiani, Phys. Rev. D 2, 1285 (1970).

[3] G. Aad et al. (ATLAS Collaboration), Phys. Lett. B 716, 1 (2012); S. Chatrchyan et al. (CMS Collaboration), Phys. Lett. B 716, 30 (2012).

[4] R. Dermisek, J. F. Gunion, and B. McElrath, Phys. Rev. D 76, 051105 (2007); R. Dermisek and J. F. Gunion, Phys. Rev. D 81, 075003 (2010).

[5] R. Dermisek and J. F. Gunion, Phys. Rev. Lett. 95, 041801 (2005); R. Dermisek, J. F. Gunion, and B. McElrath, Phys. Rev. D 76, 051105 (2007).

[6] F. Wilczek, Phys. Rev. Lett. 39, 1304 (1977).

[7] M. Lisanti and J. G. Wacker, Phys. Rev. D 79, 115006 (2009).

[8] B. Aubert et al. (BABAR Collaboration), Phys. Rev. Lett. 103, 081803 (2009).

[9] B. Aubert et al. (BABAR Collaboration), Phys. Rev. Lett. 103, 181801 (2009).

[10] P. del Amo Sanchez et al. (BABAR Collaboration), Phys. Rev. Lett. 107, 021804 (2011).

[11] J. P. Lees et al. (BABAR Collaboration), Phys. Rev. Lett. 107, 221803 (2011).

[12] W. Love et al. (CLEO Collaboration), Phys. Rev. Lett. 101, 151802 (2008).
[13] B. Aubert et al. (BABAR Collaboration), Nucl. Instrum. Methods Phys. Res., Sect. A 479, 1 (2002).

[14] D. Lange, Nucl. Instrum. Methods Phys. Res., Sect. A 462, 152 (2001).

[15] S. Agostinelli et al. (Geant4 Collaboration), Nucl. Instrum. Methods Phys. Res., Sect. A 506, 250 (2003).

[16] K. Nakamura et al. (Particle Data Group), J. Phys. G 37, 075021 (2010).

[17] W. Menges, in Nuclear Science Symposium Conference Record (IEEE, New York, 2006), Vol. 5, p. 1470.

[18] A. Höcker et al., Proc. Sci., ACAT (2007) 040; http:// tmva.sourceforge.net/.

[19] B. Aubert et al. (BABAR Collaboration), Phys. Rev. Lett. 103, 251801 (2009).

[20] G. Punzi, arXiv:physics/0308063.

[21] J. P. Lees et al. (BABAR Collaboration), Phys. Rev. D 84, 092003 (2011).

[22] M. J. Oreglia, Ph.D. Thesis, Stanford University [Report No. SLAC-236, 1980], Appendix D.

[23] B.H. Hooberman, Ph.D. Thesis, UC Berkeley [Report No. SLAC-R-924, 2009].

[24] M. L. Mangano and P. Nason, Mod. Phys. Lett. A 22, 1373 (2007).

[25] P. Nason, Phys. Lett. B 175, 223 (1986).

[26] R. Barbieri, R. Gatto, R. Kögerler, and Z. Kunszt, Phys. Lett. 57B, 455 (1975).

[27] M. Beneke, A. Signer, and V. A. Smirnov, Phys. Rev. Lett. 80, 2535 (1998). 